\title{
ON THE POINT SPECTRUM OF POSITIVE OPERATORS
}

\author{
HELMUT H. SCHAEFER ${ }^{1}$
}

1. Recently, G.-C. Rota proved the following result:

Let $(S, \Sigma, \mu)$ be a measure space of finite measure, $P$ a positive linear operator on $L_{1}(S, \Sigma, \mu)$ with $L_{1}$-norm and $L_{\infty}$-norm at most one. If $\alpha,|\alpha|=1$, is an eigenvalue of $P$ such that $\alpha f=P f\left(f \in L_{1}\right)$, then $\alpha^{2}$ is an eigenvalue such that $\alpha^{2}|f| g^{2}=P\left(|f| g^{2}\right)$, where $f=|f| g$.

It can be added that $\alpha^{n}|f| g^{n}=P\left(|f| g^{n}\right)$ for every integer $n$; thus Rota proved for a fairly large class of operators, without compactness assumptions, a result known (and due to Frobenius) for positive finite square matrices, and known for certain types of positive operators under conditions guaranteeing that the spectrum intersects the circumference of the spectral circle but in a finite set (see Karlin [ 1 , pp. 933-935] for an excellent survey and some more general examples). Simple but typical examples of operators showing the spectral behavior exhibited in Rota's theorem are the permutation matrices on $l_{p}(1 \leqq p \leqq \infty)$.

The purpose of this paper is to extend Rota's result to a larger class of spaces and operators. Apart from the particular type of underlying space, the stringent condition in Rota's theorem (supposing that $\mu(S)=1)$ is $r(T)=\|T\|_{1}=\|T\|_{\infty}, r(T)$ denoting the spectral radius of $T$ in $L_{1}$ which is implicitly assumed to be one in [2]. From this, we can drop the total finiteness of $\mu$, the assumption $\|T\|_{1}=\|T\|_{\infty}$ and the requirement that $L_{\infty}$ be invariant under $T$ ( $T$ need indeed not be defined on all of $L_{\infty}$ when $\mu(S)$ is infinite). More generally (Theorem 1), the result is true for positive operators on any complex function space $E$ of type $L_{p}(S, \Sigma, \mu)$ or $C(X)$ (X compact Hausdorff), whenever $T^{\prime} \psi \leqq \psi$ for some strictly positive linear form $\psi \in E^{\prime} .^{2}$ This class includes all quasi-interior positive operators on $C(X)$, for which other spectral properties were obtained in [3]. More particularly, for positive matrix operators on $l_{p}$ satisfying the assumption above with respect to some strictly positive linear form, the presence (assuming $r(T)=1$ ) of a single unimodular eigenvalue which is not a root of unity, implies that the entire unit circle is in the point spectrum of $T$ (Theorem 2).

The assumption that $T^{\prime} \psi \leqq \psi$ for some strictly positive linear form, in particular satisfied through $\|T\|_{1}=1$ in Rota's theorem, is by no means necessary for the conclusion; it is made to ensure that

Received by the editors September 26, 1962.

1 Work sponsored by the U.S. Army Research Office.

2 $E^{\prime}$ denotes the (topological) dual of $E, T^{\prime}$ the adjoint of $T$. 
$\alpha f=T f(|\alpha|=1)$ implies $|f|=T|f|$. To see how close this comes to what may be needed, we remark that every permutation matrix is the direct sum of a (possibly infinite) number of summands satisfying that assumption on a $T$-reducing subspace of $l_{p}$.

2. In this section, we formulate the results and their immediate consequences. By $(S, \Sigma, \mu)$ we understand a measure space of finite or infinite measure, and by $C(X)$ the $B$-space (under the sup-norm) of all continuous complex functions on a compact (Hausdorff) space $X$. By a space of type $C(X)$ we mean an ordered complex $B$-space which is (simultaneously algebraically, topologically, and order) isomorphic to some $C(X)$ (but not necessarily isometric), and likewise for $L_{p}(S, \Sigma, \mu)(1 \leqq p<\infty)$. $J$ denotes the set of all rational integers.

TheOREM 1. Let $E$ be a space of type $L_{p}(S, \Sigma, \mu)(1 \leqq p<\infty)$ or $C(X)$, and let $T$ be a positive linear operator on $E$, with spectral radius 1 , such that $T^{\prime} \psi \leqq \psi$ for some strictly positive linear form on $E$. Then:

If $\alpha f=T f,|\alpha|=1$, then $\alpha^{n}|f| g^{n}=T\left(|f| g^{n}\right)$ for every $n \in J$, where $f=|f| g$.

COROLLARY 1. Under the conditions of the theorem, the point spectrum of $T$ on the unit circle consists either of a finite ${ }^{3}$ number of (groups of) roots of unity, or it is dense.

It is not difficult to see that Theorem 1 contains Rota's theorem as a special case. In fact, if $\|T\|_{1} \leqq 1$, then $T^{\prime} \psi \leqq \psi$ (and conversely), where $\psi: f \rightarrow \int f d \mu$ is a strictly positive linear form on $L_{1}(S, \Sigma, \mu)$.

There is another class of positive operators satisfying the condition of Theorem 1. A positive operator $T$ on an ordered $B$-space $E$ is called quasi-interior [3] if there exists some $\lambda_{0}>r(T)$ such that $T R\left(\lambda_{0}\right) x$ is a quasi-interior point of the positive cone $K$ of $E$, for each $0 \neq x \in K$; $x_{0} \in K$ is quasi-interior if the order interval $\left[0, x_{0}\right]$ is a total subset of $E$. (For more details on quasi-interior maps, see [3].)

CoROLlaRY 2. The assertion of Theorem 1 is valid for every quasiinterior positive map on a space of type $C(X)$.

Proof. It is known (see, e.g., [3, Theorem 1, Corollary]) that for any positive operator on $C(X)$ with spectral radius $r$, there exists a nonzero linear form $\psi \geqq 0$ satisfying $r \psi=T^{\prime} \psi$. Since for every $f \in C(X)$, and $\lambda>r$,

$$
\psi(f) \sum_{n=1}^{\infty} \frac{r^{n}}{\lambda^{n}}=\psi[T R(\lambda) f],
$$

3 This is meant to include the case of empty point spectrum on $|\lambda|=1$. 
it follows that $\psi$ is strictly positive when $T$ is quasi-interior and $r>0$.

If $T$ is an operator on a space $l_{p}(1 \leqq p \leqq \infty)$, represented by a positive matrix, we obtain the following stronger result.

THEOREM 2. Let $T$ be a positive matrix operator on some space $l_{p}(1 \leqq p \leqq \infty)$, satisfying the assumptions of Theorem 1 . If $T$ has $a$ unimodular eigenvalue which is not a root of unity, then the entire unit circle belongs to the point spectrum of $T$.

On the other hand, it results from the proof of Theorem 2 that if such a matrix has strictly positive diagonal entries, its point spectrum on the unit circle can at most contain the number 1.

3. The proof of Theorems 1 and 2 is divided into several steps. Unless any further distinction is needed, we denote by $E$ any Banach space of the type considered in Theorem 1.

(a) For any $f \in E$ and positive linear operator $T$ on $E,|T f| \leqq T|f|$. Let $s$ be a fixed element of $S$ (or $X$, respectively). We have $|T f|(s)$ $=(T \tilde{f})(s)$ where $\tilde{f}=f e^{i \delta}$ and $\delta=\delta(s)$ is suitably chosen. Let $\tilde{f}=\tilde{g}+i \tilde{h}$, $\tilde{g}$ and $\tilde{h}$ denoting the real and imaginary parts of $\tilde{f}$, respectively. Now $T \tilde{f}=T \tilde{g}+i T \tilde{h}$, and $T \tilde{h}(s)=0$ since $T \tilde{g}, T \tilde{h}$ are real-valued elements of $E$. Hence we have

$$
T \tilde{f}(s)=T \tilde{g}(s) \leqq T|\tilde{g}|(s) \leqq T|f|(s)
$$

since $|\tilde{g}| \leqq|\tilde{f}|=|f|$, whence it follows that $|T f|(s) \leqq T|f|(s)$. $s$ being arbitrary, we conclude that $|T f| \leqq T|f|$.

(b) If $\alpha f=T f$ where $0 \neq f \in E$ and $|\alpha|=1$, it follows that $|f|=|T f|$ and hence, by (a), that $|f| \leqq T|f|$. By assumption, there exists a strictly positive linear form satisfying $T^{\prime} \psi \leqq \psi$; hence $\psi(|f|) \leqq \psi(T|f|)$ $=T^{\prime} \psi(|f|) \leqq \psi(|f|)$ and, therefore, $\psi(T|f|-|f|)=0$. Since $\psi$ is strictly positive, $|f|=T|f|$.

(c) Let $H_{0}=\{t:|f(t)|>0\}$, and let $F$ denote the vector subspace of $E$ whose elements are of the form $|f| g, g \in G$, where $G$ is the vector space of all bounded $\Sigma$-measurable or all bounded continuous functions (accordingly as $E=L_{p}(S, \Sigma, \mu)$ or $E=C(X)$ ), on $H_{0}$. Since $|T| f|g| \leqq\|g\|_{\infty} T|f|, F$ is invariant under $T$. The formula

$$
(U g)(s)=|f(s)|^{-1}\{T|f| g\}(s) \quad\left(s \in H_{0}\right)
$$

defines a positive endomorphism of $G$. Endowed with the sup-norm, $G$ is isomorphic (even isometric) with a space $C(Y), Y$ compact. This is clear when $E=L_{p}$ (more precisely, one will consider the quotient space of $G$ modulo $\mu$-null functions), from the Gel'fand-Naimark theorem; the same conclusion holds when $E=C(X)$, but here, more concretely, $G$ is isomorphic with $C(Y)$, where $Y$ is the Stone-Cech 
compactification of $H_{0} \subset X$. Thus in any case, we can associate with $f$, satisfying $|f|=T|f|$, a positive operator $U$ on $C(Y)$ such that $U e=e(e$ the constantly-one function on $Y$ ), and hence $\|U\|=1$.

(d) We write $f=|f| g$, where $g$ is well defined on $H_{0}$; moreover, $|g|=e$ and $\alpha g=U g$ since $\alpha f=T f$ by assumption. We identify $G$ and $C(Y)$ in the spirit of the preceding paragraph. For each $s \in Y$, the mapping $h \rightarrow(U h)(s)$ defines a positive Radon measure $m_{s}$, of mass 1 , on $Y$. The remainder of the proof rests on the following lemma.

Lemma. Let $g \in C(Y)$ satisfy $|g|=e$, and let $s \in Y$ be arbitrary. If $\alpha g=U g,|\alpha|=1$, then the support of $m_{s}$ is contained in $\{t \in Y: g(t)$ $=\alpha g(s)\}$.

The proof of this lemma is elementary, and will be omitted. Now let $n$ be any integer. Since for fixed $s, g(t)=\alpha g(s)$ on the support of

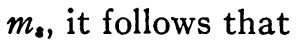

$$
\left(U g^{n}\right)(s)=\int g^{n}(t) d m_{s}(t)=\alpha^{n} g^{n}(s)
$$

$s \in Y$, which proves that $\alpha^{n} g^{n}=U g^{n}$. Translating back into $E$, we obtain $\alpha^{n}|f| g^{n}=T\left(|f| g^{n}\right)$, completing the proof of Theorem 1.

(e) It remains to prove Theorem 2. Let $A=\left(a_{i k}\right)$ be the matrix representing the operator $T$, and let $\alpha x=A x$ where $x \neq 0$ is a vector in $l_{p}$ and $\alpha,|\alpha|=1$, is not a root of unity. It follows from Theorem 1 that $|x|=A|x|$. As in (c) above, we associate with $A$ an operator $U$ on $l_{\infty}\left(H_{0}\right)$ where $H_{0}$ is the set of subscripts on which the coordinates of $x$ are nonzero; more precisely, $U$ is represented by the matrix $\left(u_{i k}\right)$, where $u_{i k}=\left|x_{i}\right|^{-1} a_{i k}\left|x_{k}\right|$ for $i, k \in H_{0}$. Let $x_{i}=\left|x_{i}\right| v_{i}$ for $i \in H_{0}$, $v=\left(v_{i}\right)$. Further, denote by $\Gamma$ a set of representatives of the quotient group of the group of unimodular complex numbers over the subgroup $\left\{\alpha^{n}: n \in J\right\}$. If $F_{n}=\left\{j \in H_{0}: v_{j} \in \alpha^{n} \Gamma\right\}, n \in J$, the $F_{n}$ are disjoint sets whose union is $H_{0}$.

From $\alpha v=U v$ it follows, as in the lemma in (d), that if $v_{i}=\tau$ then $v_{j}=\alpha \tau$ for all $j$ such that $u_{i j}>0\left(i, j \in H_{0}\right)$. (In fact, the lemma can be formally applied since $v \rightarrow(U v)_{i}$ is a Baire measure on the locally compact, discrete space $\left.H_{0}.\right)^{4}$ Denoting by $x^{(n)}$ the "characteristic" vector of $F_{n}$ in $l_{\infty}\left(H_{0}\right)$, we conclude that $U x^{(n+1)}=x^{(n)}$. Moreover, the $x^{(n)}$ are mutually disjoint and $\sum\left\{x^{(n)}: n \in J\right\}=e$, the constantlyone function on $H_{0}$.

Now let $\beta$ be any unimodular complex number. Letting

$$
w=\sum_{n \in J} \beta^{n} x^{(n)},
$$

- At this point the assumption that $T$ be a matrix operator is essentially used. 
it follows that $U w=\beta w$, hence $\beta$ is in the point spectrum of $U$. Set $y=\left(y_{i}\right)$, where $y_{i}=\left|x_{i}\right| w_{i}$ for $i \in H_{0}$ and $y_{i}=0$ for $i \notin H_{0}$. It is immediate that $T y=\beta y$ whence the theorem follows.

\section{REFERENCES}

1. S. Karlin, Positive operators, J. Math. Mech. 8 (1959), 907-937.

2. G.-C. Rota, On the eigenvalues of positive operators, Bull. Amer. Math. Soc. 67 (1961), 556-558.

3. H. H. Schaefer, Some spectral properties of positive linear operators, Pacific J. Math. 10 (1960), 1009-1019.

UNIVERSITY OF MICHIGAN 